\title{
The Analysis of ETM Image's Spectral and Its Implication on Water Depth Inversion in Xiamen Bay
}

\author{
Xiaodong Zhang \\ College of Harbor Coastal and Offshore Engineering \\ Hohai University \\ Nanjing, China \\ Wenhua Zeng \\ Institute of Meteorology and Marine \\ PLA University of Science and Technology \\ Nanjing, China
}

\author{
Shouxian Zhu \\ College of Harbor Coastal and Offshore Engineering \\ Hohai University \\ Nanjing, China \\ zhushouxian@vip.sina.com
}

Wenjing Zhang

Institute of Meteorology and MarineCollege

PLA University of Science and Technology

Nanjing, China

\begin{abstract}
Dual-spectra ratio method is generally considered superior to single-spectra approach in the most previous studies for water depth inversion by ETM image. In this paper, the correlation between the ETM's spectral and the water depth is analyzed. TM2 gives the largest absolute correlation index in all the single-spectra and dual-spectral ratio. Then some models using linear, logarithmic, power exponential and exponential equations have been made for water depth inversion. The exponential model based on TM2 is proved to be the best one, and it brings error of $24.6 \%$.
\end{abstract}

Index Terms-Water depth inversion; Remote sensing; ETM; Xiamen Bay

\section{INTRODUCTION}

Explorations in water depth inversion using remote sensing data have begun to be done since the launch of the first Landsat satellite in 1972. it has been widely used for port and waterway engineering research and riverbed evolution analysis because of convenience and economy ${ }^{[1-7]}$.It is based on the theoretical basis that : (1)The visible light has good ability to penetrate water; (2) The light radiation intensity transmitting to the water is gradually weakened because of the absorption and reflection effect of the water ${ }^{[2,7]}$. However, the remote sensing visible spectra are also affected by other factors such as sediment composition, suspended sediment, water quality and atmospheric conditions ${ }^{[8]}$.

Jerlov has carried out depth inversion study using a singleband model, in which the seabed reflection and water decay coefficient were taken as uniform in the study area ${ }^{[9]}$. Poleyn and Sattinger proposed the band ratio method to eliminate the influence of varied seabed reflection and decay coefficient of the water ${ }^{[10]}$. Paredes and Spero studied water depth inversion using multi-band method for many types of seabed sediment ${ }^{[11]}$ In the most previous studies for water depth inversion by ETM image, dual-spectra ratio method is generally considered superior to single- spectra approach ${ }^{[10,12,13,14]}$.
The purpose of this paper is to analyze the character of ETM Image's Spectral and make depth inversion in Xiamen Bay.

\section{THE STUDY AREA AND DATE}

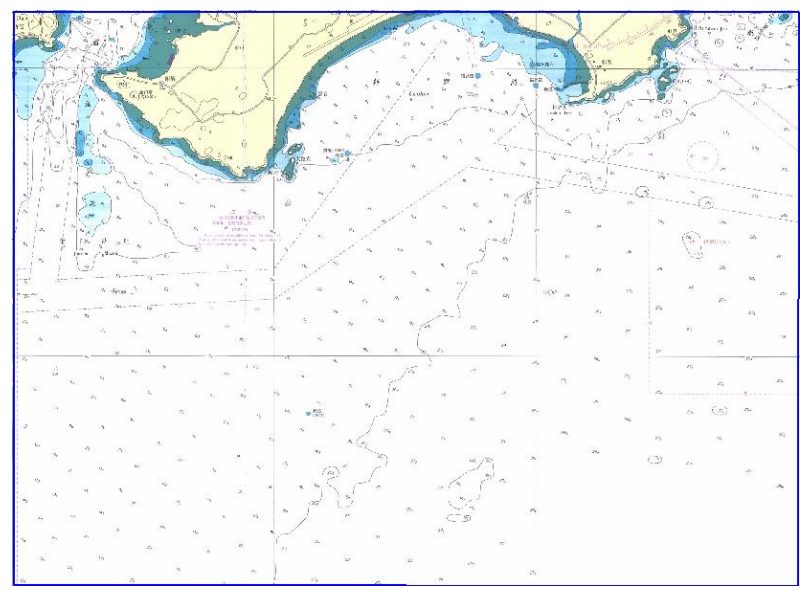

Fig. 1. The study area

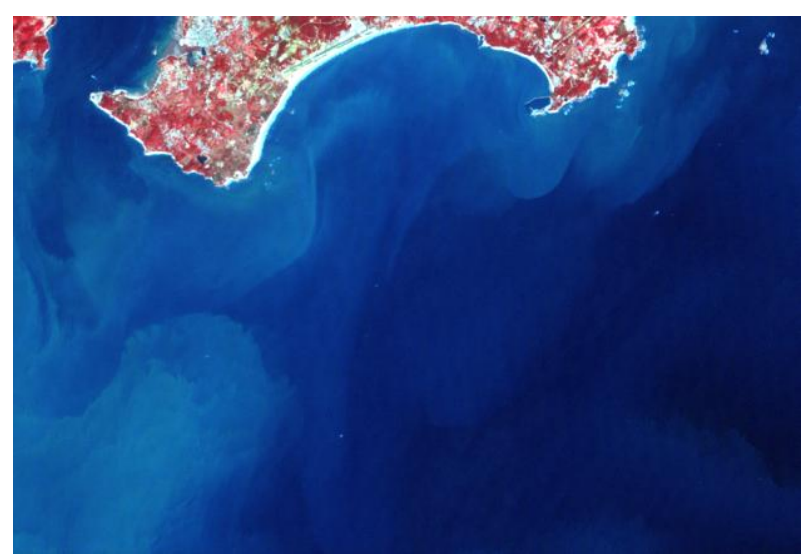

Fig. 2. The remote sensing image 
The study area is $118^{\circ} 15^{\prime} \mathrm{E} \sim 118^{\circ} 30^{\prime} \mathrm{E}$ and $24^{\circ} 16^{\prime}$ $\mathrm{N} \sim 24^{\circ} 26^{\prime} \mathrm{N}$. The observed depth data for depth inversion is from the underwater topographic map of Xiamen Bay published in 2001. The study area is shown in Figure 1. There are 553 sample points of observed depth data in the study area.

Remote sensing data is from Landsat-7 satellite image on March 7, 2002. The remote sensing image is shown in Figure 2. It is processed by radiometric calibration operation and FLAASH atmospheric correction.

\section{SPECTRAL ANALYSIS}

There are six bands in TM visible light infrared group. Two of them are short-wave infrared bands, which have bad ability to penetrate water. So we only analyze the correlation between the other four bands and the observed depth. The correlation equation $^{[13]}$ is shown as follows. In Equation $1, r$ is the correlation index, $\mathrm{x}_{\mathrm{i}}$ is the reflectance factor, $\overline{\mathrm{x}}$ is the average of the reflectance factor, $y_{i}$ is water depth, $\bar{y}$ is the average of water depth, $\mathrm{N}$ is the number of samples. The correlation index of reflectivity factor and depth value is shown in Table 1 . In the four single bands, namely TM1, TM2, TM3 and TM4, TM2 has the best correlation with the observed water depth data. TM1/TM3 gives the larger correlation index than other ratios of two spectra such as TM1/TM2, TM1/TM4, TM2/TM3, TM2/TM4 and TM3/TM4. TM2 gives the larger correlation index than TM1/TM3.

$$
r=\frac{\sum_{i=1}^{N}\left(x_{i}-\bar{x}\right)\left(y_{i}-\bar{y}\right)}{\sqrt{\sum_{i=1}^{N}\left(x_{i}-\bar{x}\right)^{2}} \sqrt{\sum_{i=1}^{N}\left(y_{i}-\bar{y}\right)^{2}}}
$$

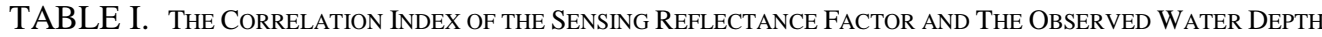

\begin{tabular}{|c|c|c|c|c|c|c|c|c|c|c|}
\hline $\mathrm{x}$ & TM1 & $\mathrm{TM} 2$ & TM3 & TM4 & TM1/TM2 & TM1/TM3 & TM1/TM4 & TM2/TM3 & TM2/TM4 & TM3/TM4 \\
\hline$r$ & -0.616 & -723 & -0.667 & -0.547 & 0.595 & 0.659 & 0.29 & 0.598 & -0.082 & -0.543 \\
\hline
\end{tabular}

\section{RESULT OF WATER DEPTH INVERSION}

The reflectance factors TM2 and TM1/TM3 are selected to found water depth inversion models with linear, logarithmic, power exponential and exponential equations. These models are checked by the observed depth data as Table 2 . The best model using TM2 is with exponential equation, its average relative error is 0.246 . The best model using TM1/TM3 is with exponential equation, its average relative error is 0.269 . Figure 3 gives the depth fitting effect with exponential equation of TM2 and TM1/TM3. Figure 4 gives the result of depth inversion with exponential equation of TM2. The inversed depth becomes shallow to shore, which accords with the main actual character.

TABLE II. THE CHECK OF THE DEPTH INVERSION MODELS

\begin{tabular}{|c|c|c|c|}
\hline $\begin{array}{c}\text { Reflectance } \\
\text { factor(x) }\end{array}$ & Model type & Equation & Mean relative error \\
\hline \multirow{4}{*}{ TM2 } & Linear & $y=-589.272 x+61.596$ & 0.350 \\
\cline { 2 - 4 } & Logarithmic & $y=0.213 x^{-1.780}$ & 0.305 \\
\cline { 2 - 4 } & Power exponential & $y=110.844 e^{-21.648 x}$ & 0.248 \\
\cline { 2 - 4 } & Exponential & $y=15.173 x-2.559$ & 0.246 \\
\cline { 2 - 4 } & Linear & $y=20.578 \ln x+12.279$ & 0.341 \\
\cline { 2 - 4 } & Logarithmic & $y=11.986 x^{1.130}$ & 0.349 \\
\cline { 2 - 4 } & Power exponential & $y=5.334 e^{0.829 x}$ & 0.273 \\
\cline { 2 - 4 } & Exponential & \multirow{2}{*}{0.269} & \\
\hline
\end{tabular}



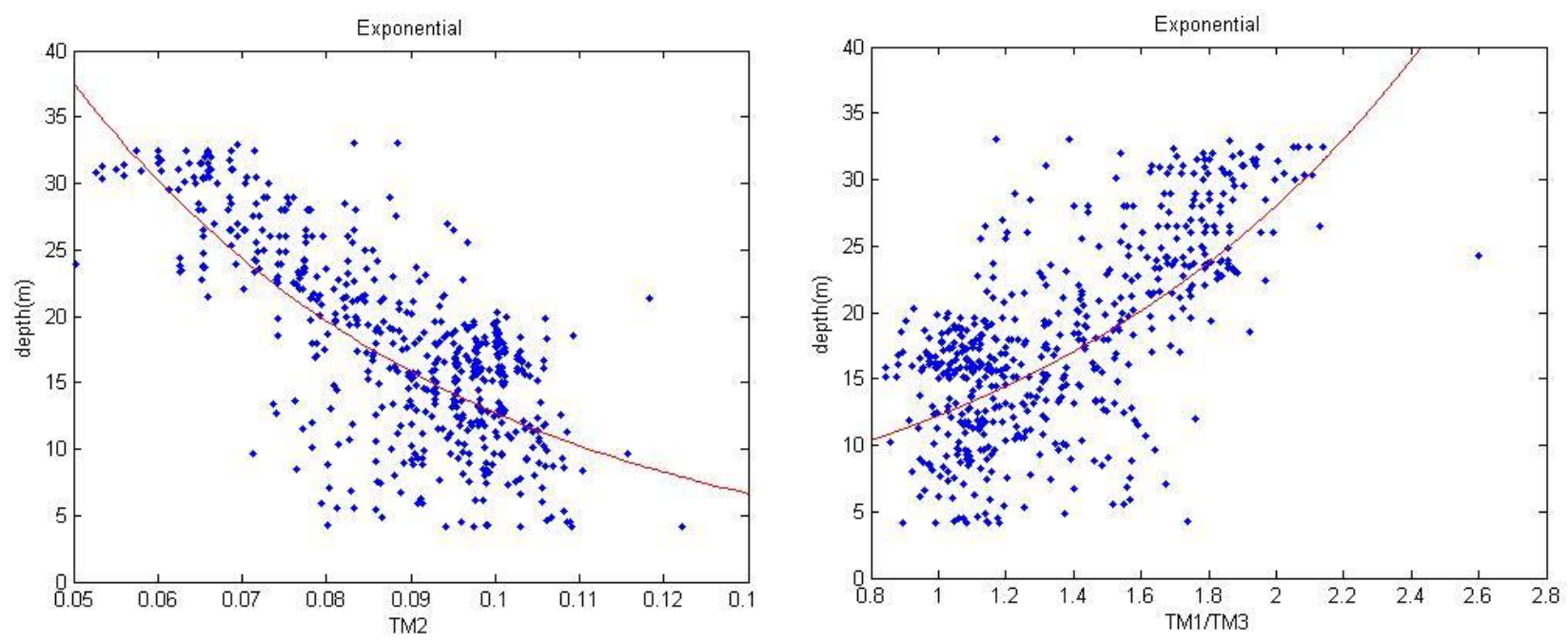

Fig. 3. The depth fitting effect with exponential equation of TM2 and TM1/TM3
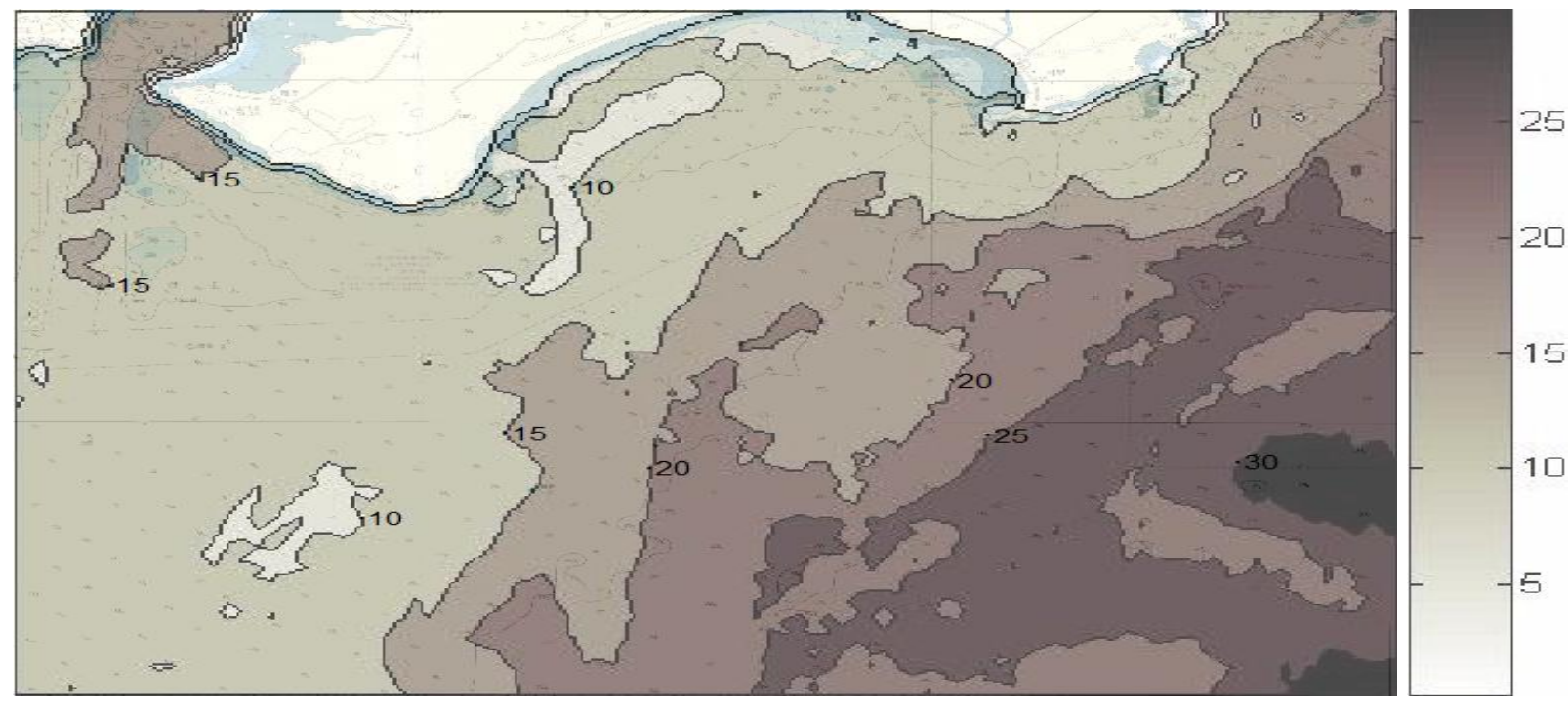

Fig. 4. The result of depth inversion with exponential equation of TM2

\section{USING THE TEMPLATE}

The spectral analysis of ETM in the south of Xiamen Bay shows that TM2 gives the largest absolute correlation index in all the single-spectra and dual-spectral ratio. The depth inversions with TM2 and TM1/TM3 show that power exponential and exponential equations bring smaller error than linear and logarithmic equations. The depth inversion can give the main character of seabed. The error of depth inversion with exponential equation of TM2 is $24.6 \%$, which is the least in the all depth inversions with TM2 and TM1/TM3. It can be proved that single-spectra may be better than dual-spectral ratio in depth version somewhere.

\section{ACKNOWLEDGMENT}

The study has been supported by the National Natural Science Foundation (No.41076048 and No.40906044) and the
Operation Expenses for Universities' Basic Scientific Research of Central Authorities (No.2011B05714). Corresponding author : Shouxian Zhu.

\section{REFERENCES}

[1] Shen Jiashuang, Shixiang Pan.Coastal bathymetry technology Method [J]. Hydrographic Surveying and Charting, 2002, 22(6):60-65.

[2] Lyzenga, D R. Passive remote sensing techniques for mapping water depth and bottom features [J]. Applied Optics, 1978,17(3):379-383.

[3] Polcyn, F C, Sattinger,I J. Water depth determiantion using remote sensing techniques[A]. Proceedings of the 6th International Symposium on Remote Sensing of Environment, Ann Arbor, Michigan[C]. 1979:583-602.

[4] Hengle, W V, Spitzer, D. Multi-temporal water depth mapping by means of Landsat TM [J]. International Journal of Remote Sensing, 1991,12(4):703-712. 
[5] Roberts A C B. Shallow water bathymetry using integrated airborne multi-spectral remote sensing [J]. Int. J. Remote Sensing, 1999,20(3):497-510.

[6] Tripathi, N K, Rao, A M. Bathymetric mapping in Kakinada Bay, India, using RS-1DLISS-III data [J]. International Journal of Remote Sensing, 2002,23(6):1013-1025.

[7] Weijiet al. Satellite remote sensing bathymetry for modeling [J]. Photogrammetric Engineering and Remote Sensing, 1992,58(5):545-549.

[8] Baban, S M J. The evaluation of different algorithms for bathymetric charting of lakes using Landsat imagery $[\mathrm{J}]$. International Journal of Remote Sensing, 1993(14):2263-2273.

[9] Jerlov, N. G. Marine Optics[M]. Elsevier Scientific, Amsterdam.

[10] Polcyn, F. C., Sattinger, I. J. Water depth determination using remote sensing techniques[A]. In Proceedings of the Sixth International Symposium on Remote Sensing of
Environment[C]. Ann Arbor, MI, 13-16 October, 1969, 10171028.

[11] Paredes, J. M., Spero, R. E. Water depth mapping from passive remote sensing data under a generalized ratio assumption[J]. Applied Optics, 1983, 22,1134-1135.

[12] Huang Jiazhu, You yuming. Experiment of water depth surveying in the Nantong section of the Yangtze River[J]. Advances in Water Science, 2002,13(2):235-238.

[13] Wang Jing-jing, Tian Qing-jiu. A research on coastal bathymetry retrieval from TM image[J]. Application Technology, 2006: 27-30.

[14] Sun Tao, Pang Zhiguo, Pan Shibing, Ge Dexiang. Study on water depth in ertan reservoir aera based on ETM remote sensing images[J]. Geography and Geo-Information Science, 2010,26(4):64 -66. 